\title{
Global existence and stability of a class of nonlinear evolution equations with hereditary memory and variable density
}

Fushan $\mathrm{Li}^{1 *}$ (D) and Zhiqiang Jia'

${ }^{\text {*Correspondence: }}$

fushan99@163.com

'School of Mathematical Sciences,

Qufu Normal University, Shandong,

P.R. China

\section{Springer}

\section{Abstract}

In this paper, we consider the initial boundary value problem of nonlinear evolution equation with hereditary memory, variable density, and external force term

$$
\left\{\begin{array}{l}
\left|u_{t}\right|^{\rho} u_{t t}-\alpha \Delta u-\Delta u_{t t}+\int_{-\infty}^{t} \mu(t-s) \Delta u(s) d s-\gamma \Delta u_{t}=f(u), \\
\quad(x, t) \in \Omega \times \mathbb{R}^{+}, \\
u(x, t)=0, \quad(x, t) \in \partial \Omega \times \mathbb{R}^{+}, \\
u(x, 0)=u_{0}(x), \quad u_{t}(x, 0)=u_{1}(x), \quad x \in \Omega .
\end{array}\right.
$$

Under suitable assumptions, we prove the existence of a global solution by means of the Galerkin method, establish the exponential stability result by using only one simple auxiliary functional, and give the polynomial stability result.

MSC: 35L05; 35L15; 35L70

Keywords: Hereditary memory; Variable density; Global existence; Exponential stability; Polynomial stability

\section{Introduction}

In this paper, we are concerned with the following problem:

$$
\left\{\begin{array}{l}
\left|u_{t}\right|^{\rho} u_{t t}-\alpha \Delta u-\Delta u_{t t}+\int_{-\infty}^{t} \mu(t-s) \Delta u(s) d s-\gamma \Delta u_{t}=f(u), \\
\quad(x, t) \in \Omega \times \mathbb{R}^{+} \\
u(x, t)=0, \quad(x, t) \in \partial \Omega \times \mathbb{R}^{+}, \\
u(x, 0)=u_{0}(x), \quad u_{t}(x, 0)=u_{1}(x), \quad x \in \Omega
\end{array}\right.
$$

where $\Omega$ is a bounded domain of $\mathbb{R}^{n}(n \geq 1)$ with smooth boundary $\partial \Omega, \rho$ is a positive constant, and $\gamma \geq 0$. We prove the existence of a global solution by means of the Galerkin method and establish the exponential stability under suitable assumptions by using a simpler auxiliary functional than that in [1]. We also show the polynomial stability under suitable conditions.

Partial differential equations in viscoelastic materials have important physical background and important mathematical significance. The viscous effects are described and

(c) The Author(s) 2019. This article is distributed under the terms of the Creative Commons Attribution 4.0 International License (http://creativecommons.org/licenses/by/4.0/), which permits unrestricted use, distribution, and reproduction in any medium, provided you give appropriate credit to the original author(s) and the source, provide a link to the Creative Commons license, and indicate if changes were made. 
characterized by an integral term, and the integral term indicates a dissipative effect. For mathematical analysis on the motions of evolution equations with memory, we refer to $[8$, 32]. Problem (1.1) is related to the equations

$$
f\left(u_{t}\right) u_{t t}-\Delta u-\Delta u_{t t}=0,
$$

which have several modeling features. If $f\left(u_{t}\right)$ is a constant, Eq. (1.2) has been used to model extensional vibrations of thin rods (see [27, Ch. 20]) and it differs from D'Alembert's wave equation because of $\Delta u_{t t}$, which is not a damping term. On the contrary, $\Delta u_{t t}$ increases the energy functional. If $f\left(u_{t}\right)$ is not a constant, Eq. (1.2) shows that the density of materials depends on the velocity $u_{t}$.

In the past ten years, several authors studied the homogeneous Dirichlet boundary value problem for the following model with memory (starting from the zero moment) and variable density:

$$
\left|u_{t}\right|^{\rho} u_{t t}-\Delta u+\int_{0}^{t} g(t-\tau) \Delta u(\tau) d \tau+F\left(u, u_{t}, u_{t t}\right)=0
$$

in a bounded domains $\Omega \subset \mathbb{R}^{n}$. Cavalcanti et al. [2] considered the model with integral dissipation and strong damping

$$
\left|u_{t}\right|^{\rho} u_{t t}-\Delta u-\Delta u_{t t}+\int_{0}^{t} g(t-s) \Delta u(s) d s-\gamma \Delta u_{t}=0, \quad(x, t) \in \Omega \times \mathbb{R}^{+} .
$$

Assuming that $0<\rho \leq \frac{2}{n-2}$ if $n \geq 3$ or $\rho>0$ if $n=1,2$ and that $g(t)$ decays exponentially, they obtained the global existence of a solution for $\gamma \geq 0$ and the uniform exponential decay of the energy for $\gamma>0$. Cavalcanti et al. [3] considered this model and proved intrinsic decays for large classes of relaxation kernels described by the inequality $g^{\prime}+H(g) \leq 0$ with convex function $H$. Han and Wang [11] considered the equation with integral dissipation and linear damping

$$
\left|u_{t}\right|^{\rho} u_{t t}-\Delta u-\Delta u_{t t}+\int_{0}^{t} g(t-s) \Delta u(s) d s+u_{t}=0, \quad(x, t) \in \Omega \times \mathbb{R}^{+} .
$$

They proved the global existence and exponential decay when $g$ is decaying exponentially by introducing two auxiliary functionals. Han and Wang [12] established the general decay of energy for the equation with integral dissipation and nonlinear damping

$$
\left|u_{t}\right|^{\rho} u_{t t}-\Delta u-\Delta u_{t t}+\int_{0}^{t} g(t-s) \Delta u(s) d s+\left|u_{t}\right|^{m} u_{t}=0, \quad(x, t) \in \Omega \times \mathbb{R}^{+},
$$

by introducing two auxiliary functionals. Messaoudi and Tatar $[29,30]$ considered the equation only with integral dissipation

$$
\left|u_{t}\right|^{\rho} u_{t t}-\Delta u-\Delta u_{t t}+\int_{0}^{t} g(t-s) \Delta u(s) d s=0, \quad(x, t) \in \Omega \times \mathbb{R}^{+} .
$$

Under some assumptions on $g$, they obtained exponential and polynomial decay rates. Messaoudi and Tatar [28] studied the equation with external force term and only with 
integral dissipation

$$
\left|u_{t}\right|^{\rho} u_{t t}-\Delta u-\Delta u_{t t}+\int_{0}^{t} g(t-s) \Delta u(s) d s=b|u|^{p-2} u, \quad(x, t) \in \Omega \times \mathbb{R}^{+} .
$$

By introducing a new functional and using potential well method they showed that there exists an appropriate set $S$ (called a stable set) such that if the initial datum is in $S$, then the solution continues to live there forever. They also showed that the solution goes to zero with an exponential or polynomial rate depending on the decay rate of the relaxation function $g$. Liu [26] considered (1.3) and proved that, for certain class of relaxation functions and certain initial data in the stable set, the decay rate of the solution energy is similar to that of the relaxation function. Conversely, for certain initial data in the unstable set, there are solutions that blow up in finite time.

Now, we list some important literature on the nonlinear evolution equation with hereditary memory and variable density. Araújo et al. [1] considered the equation with integral dissipation in infinite interval

$$
\left|u_{t}\right|^{\rho} u_{t t}-\alpha \Delta u-\Delta u_{t t}+\int_{-\infty}^{t} \mu(t-s) \Delta u(s) d s-\gamma \Delta u_{t}+f(u)=h, \quad(x, t) \in \Omega \times \mathbb{R}^{+},
$$

where $\Omega$ is a bounded domain of $\mathbb{R}^{n}(n \geq 1)$ with smooth boundary $\partial \Omega$. They established the uniqueness of the solution, exponential decay, and global attractors. However, the existence of a solution is not given in detail, two auxiliary functionals are introduced to prove the exponential decay result, and the polynomial decay result is not given. Conti et al. [5] established an existence, uniqueness, and continuous dependence result for weak solutions to the nonlinear viscoelastic equation with hereditary memory on a bounded three-dimensional domain

$$
\left|\partial_{t} u\right|^{\rho} \partial_{t t} u-\Delta \partial_{t t} u+\gamma(-\Delta)^{\theta} \partial_{t} u-\alpha \Delta u+\int_{0}^{\infty} \mu(s) \Delta u(t-s) d s+f(u)=h
$$

with Dirichlet boundary conditions. In particular, the parameter $\rho$ belongs to the interval $[0,4]$, the value 4 is critical for the Sobolev embeddings, whereas $f$ can reach the critical polynomial order 5. Lately, Conti et al. [4] studied the nonlinear viscoelastic equation

$$
\left|\partial_{t} u\right|^{\rho} \partial_{t t} u-\Delta \partial_{t t} u-\Delta u+\int_{0}^{\infty} \mu(s) \Delta u(t-s) d s+f(u)=h
$$

and showed that the sole weak dissipation given by the memory term is enough to ensure the existence and optimal regularity of the global attractor $\mathcal{A}_{\rho}$ for $\rho<4$ and critical nonlinearity $f$.

In recent years, Fatori et al. [9] studied long-time behavior of a class of thermoelastic plates with nonlinear strain and long memory; the main result establishes the existence of global and exponential attractors for the strongly damped problem through a stabilizability inequality. In addition, for the weakly damped problem, they establish the exponential stability of its Galerkin semiflows. Li et al. [13-15] proved the existence uniqueness, uniform energy decay rates, and limit behavior of the solution to the nonlinear viscoelastic Marguerre-von Kármán shallow shells system. The global existence uniqueness and decay estimates for nonlinear viscoelastic equation with boundary dissipation were given in 
$[16,17,19,22-25]$. The authors in $[10,18,20,21]$ studied the blowup phenomenon for some evolution equations. $\mathrm{Du}$ and $\mathrm{Li}[6,7]$ proved the integrability and regularity of the solution to some equations.

In this paper, we study the equation with hereditary memory $\left(u_{0}(x, t), t \leq 0\right)$ and variable density

$$
\left|u_{t}\right|^{\rho} u_{t t}-\alpha \Delta u-\Delta u_{t t}+\int_{-\infty}^{t} \mu(t-\tau) \Delta u(\tau) d \tau-\gamma \Delta u_{t}=f(u)
$$

that is,

$$
\left|u_{t}\right|^{\rho} u_{t t}-\alpha \Delta u-\Delta u_{t t}+\int_{0}^{\infty} \mu(\tau) \Delta u(t-\tau) d \tau-\gamma \Delta u_{t}=f(u)
$$

which can be rewritten as

$$
\begin{aligned}
& \left|u_{t}\right|^{\rho} u_{t t}-\left(\alpha-\int_{0}^{\infty} \mu(\tau) d \tau\right) \Delta u-\Delta u_{t t} \\
& \quad-\int_{0}^{\infty} \mu(\tau) \Delta(u(x, t)-u(x, t-\tau)) d \tau-\gamma \Delta u_{t}=f(u) .
\end{aligned}
$$

This equation inspires us to define

$$
\eta:=\eta(x, t, \tau)=u(x, t)-u(x, t-\tau), \quad(x, \tau) \in \Omega \times \mathbb{R}^{+}, t \geq 0,
$$

which implies

$$
\eta_{t}(x, t, \tau)=u_{t}(x, t)-u_{t}(x, t, \tau)=u_{t}(x, t)-\eta_{\tau}(x, t-\tau), \quad(x, \tau) \in \Omega \times \mathbb{R}^{+}, t \geq 0
$$

and

$$
t=0: \eta(x, 0, \tau)=u_{0}(x, 0)-u_{0}(x,-\tau), \quad(x, \tau) \in \Omega \times \mathbb{R}^{+} .
$$

Hence Eq. (1.4) can be rewritten as

$$
\left|u_{t}\right|^{\rho} u_{t t}-\left(\alpha-\int_{0}^{\infty} \mu(\tau) d \tau\right) \Delta u-\Delta u_{t t}-\int_{0}^{\infty} \mu(\tau) \Delta \eta(\tau) d \tau-\gamma \Delta u_{t}=f(u)
$$

Without loss of generality, we assume that $\alpha-\int_{0}^{\infty} \mu(\tau) d \tau=1$. Then

$$
\left\{\begin{array}{l}
\left|u_{t}\right|^{\rho} u_{t t}-\Delta u-\Delta u_{t t}-\int_{0}^{\infty} \mu(\tau) \Delta \eta d \tau-\gamma \Delta u_{t}=f(u), \quad(x, t) \in \Omega \times \mathbb{R}^{+} \\
\eta_{t}(x, t, \tau)=u_{t}(x, t)-\eta_{\tau}(x, t, \tau) \\
u(x, 0)=u_{0}(x), \quad u_{t}(x, 0)=u_{1}(x), \\
\eta(x, t, 0)=0, \quad \eta(x, 0, \tau)=\eta_{0}(x, \tau), \\
u=0 \quad \partial \Omega \times \mathbb{R}^{+}, \quad \eta=0 \quad \partial \Omega \times \mathbb{R}^{+} \times \mathbb{R}^{+}
\end{array}\right.
$$

where

$$
\eta_{0}(x, \tau)=u_{0}(x, 0)-u_{0}(x,-\tau), \quad(x, \tau) \in \Omega \times \mathbb{R}^{+} .
$$


The main contribution of this paper are: (a) the equation with hereditary memory, variable density, and external force term is representative; (b) the detailed construction process of the energy functional is given by an integration method; (c) we give a detailed proof of the existence for the solution; (d) the proof of the exponential decay result is simplified by introducing only one auxiliary functional; (e) the polynomial decay result is established.

The outline of this paper is as follows. In Sect. 2, we present the preliminaries and important our results. In Sects. 3-5, we prove the main Theorems 2.1-2.3, respectively.

\section{Assumptions and the main results}

In this paper, we assume that the following conditions $\left(A_{1}\right)-\left(A_{3}\right)$ hold:

$\left(A_{1}\right)$

$$
0<\rho \leq \frac{2}{n-2} \quad \text { if } n \geq 3 ; \quad \rho>0 \quad \text { if } n=1,2
$$

which implies that

$$
H_{0}^{1}(\Omega) \hookrightarrow L^{2(\rho+1)}(\Omega)
$$

$\left(A_{2}\right) f: \mathbb{R} \rightarrow \mathbb{R}$ and satisfies

$$
|f(u)-f(v)| \leq c_{0}\left(1+|u|^{p}+|v|^{p}\right)|u-v|, \quad u, v \in \mathbb{R},
$$

where $c_{0}>0$ and

$$
0<p \leq \frac{2}{n-2} \quad \text { if } n \geq 3 ; \quad \rho>0 \quad \text { if } n=1,2
$$

and

$$
f(s) s \leq F(s) \leq 0, \quad \forall s \in \mathbb{R},
$$

where $F(z)=\int_{0}^{z} f(\sigma) d \sigma$.

$\left(A_{3}\right) \mu$ satisfies

$$
\mu \in C^{1}\left(\mathbb{R}^{+}\right) \cap L^{1}\left(\mathbb{R}^{+}\right), \quad 0 \leq \mu(\tau)<\infty, \quad \mu(0)>0, \quad \mu(+\infty)=0
$$

with

$$
\int_{0}^{\infty} \mu(\tau) d \tau=: k_{0}>0
$$

and there exists a constant $k_{1}>0$ satisfying

$$
\mu^{\prime}(t) \leq-k_{1} \mu^{q}(t), \quad \forall t \in \mathbb{R}^{+}, 1 \leq q<\frac{3}{2}
$$

To consider the relative displacement $\eta$ as a new function, we introduce the weighted $L^{2}$-space

$$
\mathcal{M}:=L_{\mu}^{2}\left(\mathbb{R}^{+} ; H_{0}^{1}(\Omega)\right)=\left\{v: \mathbb{R}^{+} \rightarrow H_{0}^{1}(\Omega) \mid \int_{0}^{\infty} \mu(\tau)\|\nabla \nu(\tau)\|_{2}^{2} d \tau<\infty\right\},
$$


which is a Hilbert space endowed with inner product

$$
(\nu, w)_{\mathcal{M}}=\int_{0}^{\infty} \mu(\tau)\left(\int_{\Omega} \nabla v(\tau) \cdot \nabla w(\tau) d x\right) d \tau
$$

and norm

$$
\|v\|_{\mathcal{M}}^{2}=\int_{0}^{\infty} \mu(\tau)\|\nabla v(\tau)\|_{2}^{2} d \tau
$$

We introduce the notation

$$
\mathcal{H}=H_{0}^{1}(\Omega) \times H_{0}^{1}(\Omega) \times \mathcal{M}
$$

\section{Remark 2.1}

(1) $H_{0}^{1}(\Omega) \hookrightarrow L^{r}(\Omega)$ with

$$
r: \begin{cases}2 \leq r \leq \frac{2 n}{n-2}, & n \geq 3 \\ \geq 2, & n=1,2\end{cases}
$$

which implies

$$
\|\varphi\|_{r} \leq B\|\varphi\|_{2}, \quad \forall \varphi \in H_{0}^{1}(\Omega)
$$

(2) From $\left(A_{2}\right)$ we can easily get $f(0)=0$.

(3) The condition $q<\frac{3}{2}$ is imposed to ensure that $\int_{0}^{\infty} \mu^{2-q}(\tau) d \tau<\infty$. In fact, assumption $\left(A_{3}\right)$ implies

$$
\mu(t) \leq \frac{C_{1}}{(1+t)^{\frac{1}{q-1}}}, \quad \frac{2-q}{q-1}>1,
$$

and therefore $\int_{0}^{\infty} \mu^{2-q}(\tau) d \tau<\infty$.

Give the initial data $\left(u_{0}, u_{1}, \eta_{0}\right) \in \mathcal{H}$, a function $\boldsymbol{z}=\left(u, u_{t}, \eta\right) \in C([0, T], \mathcal{H})$ is a weak solution of problem (1.5) if it satisfies the initial condition $\boldsymbol{z}(0)=\left(u_{0}, u_{1}, \eta_{0}\right)$ and

$$
\begin{aligned}
& \left(\left|u_{t}\right|^{\rho} u_{t t}, w\right)+(\nabla u, \nabla w)+\left(\nabla u_{t t}, \nabla w\right)+\gamma\left(\nabla u_{t}, \nabla w\right)+\int_{0}^{\infty} \mu(\tau)(\nabla \eta, \nabla w) d \tau \\
& \quad=(f(u), w), \\
& \left(\partial_{t} \eta+\partial_{\tau} \eta, v\right)_{\mathcal{M}}=\left(u_{t}, v\right)_{\mathcal{M}},
\end{aligned}
$$

for all $w \in H_{0}^{1}(\Omega), v \in \mathcal{M}$, and a.e. $t \in[0, T]$.

Multiplying both sides of Eq. (1.5) by $u_{t}$, integrating the resulting equation over $\Omega$, and using the Green formula, we have

$$
\begin{aligned}
& \int_{\Omega}\left|u_{t}\right|^{\rho} u_{t t} u_{t} d x+\int_{\Omega} \nabla u \cdot \nabla u_{t} d x+\int_{\Omega} \nabla u_{t t} \nabla u_{t} d x+\int_{\Omega} \nabla u_{t} \int_{0}^{\infty} \mu(\tau) \nabla \eta(\tau) d \tau d x \\
& \quad+\gamma \int_{\Omega}\left|\nabla u_{t}\right|^{2} d x=\int_{\Omega} f(u) u_{t} d x
\end{aligned}
$$


that is,

$$
\begin{aligned}
\frac{d}{d t} & {\left[\frac{1}{\rho+2}\left\|u_{t}\right\|_{\rho+2}^{\rho+2}+\frac{1}{2}\|\nabla u\|_{2}^{2}+\frac{1}{2}\left\|\nabla u_{t}(t)\right\|_{2}^{2}-\int_{\Omega} F(u) d x\right] } \\
& +\gamma\left\|\nabla u_{t}\right\|_{2}^{2}+\int_{\Omega} \nabla u_{t} \int_{0}^{\infty} \mu(\tau) \nabla \eta d \tau d x=0 .
\end{aligned}
$$

A direct computation and application of (1.5) show that

$$
\begin{aligned}
\int_{\Omega} \nabla u_{t} \int_{0}^{\infty} \mu \nabla \eta(\tau) d \tau d x & =\int_{\Omega}\left(\nabla \eta_{t}+\nabla \eta_{\tau}\right) \int_{0}^{\infty} \mu(\tau) \nabla \eta d \tau d x \\
& =\int_{\Omega} \nabla \eta_{t} \int_{0}^{\infty} \mu(\tau) \nabla \eta d \tau d x+\int_{\Omega} \nabla \eta_{\tau} \int_{0}^{\infty} \mu(\tau) \nabla \eta d \tau d x \\
& =\frac{1}{2} \frac{d}{d t}\|\nabla \eta\|_{\mathcal{M}}^{2}+\left(\nabla \eta_{\tau}, \nabla \eta\right)_{\mathcal{M}} .
\end{aligned}
$$

This computation inspires us to define an energy functional as follows:

$$
E(t)=\frac{1}{\rho+2}\left\|u_{t}\right\|_{\rho+2}^{\rho+2}+\frac{1}{2}\|\nabla u\|_{2}^{2}+\frac{1}{2}\left\|\nabla u_{t}\right\|_{2}^{2}+\frac{1}{2}\|\nabla \eta\|_{\mathcal{M}}^{2}-\int_{\Omega} F(u) d x
$$

and

$$
E^{\prime}(t)=-\gamma\left\|\nabla u_{t}\right\|_{2}^{2}-\left(\eta_{\tau}, \eta\right)_{\mathcal{M}}
$$

Using $\left(A_{3}\right)$ and (1.5), we have

$$
\left(\eta_{\tau}, \eta\right)_{\mathcal{M}}=\frac{1}{2} \int_{\Omega}\left(\int_{0}^{\infty} \mu(\tau) \frac{\partial}{\partial \tau}|\nabla \eta|^{2} d \tau\right) d x=-\frac{1}{2} \int_{\Omega}\left(\int_{0}^{\infty} \mu^{\prime}(\tau)|\nabla \eta|^{2} d \tau\right) d x
$$

Then

$$
E^{\prime}(t)=-\gamma\left\|\nabla u_{t}(t)\right\|_{2}^{2}+\frac{1}{2} \int_{0}^{\infty} \mu^{\prime}(\tau)\|\nabla \eta(\tau)\|_{2}^{2} d \tau \leq 0 .
$$

Theorem 2.1 Assume that conditions $\left(A_{1}\right)-\left(A_{3}\right)$ hold and $\gamma \geq 0$. If the initial data $\left(u_{0}, u_{1}, \eta_{0}\right) \in \mathcal{H}$, then for any $T>0$, problem (1.5) has a weak solution

$$
\left(u, u_{t}, \eta\right) \in C([0, T], \mathcal{H})
$$

satisfying

$$
\begin{aligned}
& u \in L^{\infty}\left(\mathbb{R}^{+} ; H_{0}^{1}(\Omega)\right), \quad u_{t} \in L^{\infty}\left(\mathbb{R}^{+} ; H_{0}^{1}(\Omega)\right), \\
& u_{t t} \in L^{2}\left([0, T] ; H_{0}^{1}(\Omega)\right), \quad \eta \in L^{2}\left(\mathbb{R}^{+} ; \mathcal{M}\right) .
\end{aligned}
$$

Theorem 2.2 Assume that conditions $\left(A_{1}\right)-\left(A_{3}\right)$ hold and $\gamma>0$. If $q=1$, then

$$
E(t) \leq K e^{-v t}, \quad t \geq 0,
$$

where $K$ and $v$ are positive constants. 
Theorem 2.3 Assume that conditions $\left(A_{1}\right)-\left(A_{3}\right)$ hold and $\gamma>0$. If $1<q<\frac{3}{2}$, then

$$
E(t) \leq K(1+t)^{-\frac{1}{2(q-1)}}, \quad t \geq 0
$$

where $K$ is a positive constant.

\section{Proof of Theorem 2.1}

We study the equation

$$
\left|u_{t}\right|^{\rho} u_{t t}-\Delta u-\Delta u_{t t}-\int_{0}^{\infty} \mu(\tau) \Delta \eta d \tau-\gamma \Delta u_{t}=f(u)
$$

Let $\left\{\omega_{j}\right\}_{j=1}^{\infty}$ be an orthogonal basis of $H_{0}^{1}$ with $\omega_{j}$ satisfying

$$
\left\{\begin{array}{l}
-\Delta \omega_{j}=\lambda_{j} \omega_{j}, \quad x \in \Omega \\
\left.\omega_{j}\right|_{\partial \Omega}=0
\end{array}\right.
$$

By normalization we have $\left\|\omega_{j}\right\|_{2}=1$ and write $V_{k}=\operatorname{span}\left\{\omega_{1}, \ldots, \omega_{k}\right\}$. For any given integer $k$, we consider the approximate solution

$$
u_{k}(t)=\sum_{j=1}^{k} c_{k}^{j}(t) \omega_{j}
$$

that satisfies

$$
\left\{\begin{array}{l}
\left(\left|u_{k t}\right|^{\rho} u_{k t t}, \omega_{j}\right)+\left(\nabla u_{k}, \nabla \omega_{j}\right)+\left(\nabla u_{k t t}, \nabla \omega_{j}\right)+\gamma\left(\nabla u_{k t}, \nabla \omega_{j}\right) \\
\quad+\int_{0}^{\infty} \mu(\tau)\left(\nabla \eta_{k}, \nabla \omega_{j}\right) d \tau=\left(f\left(u_{k}\right), \omega_{j}\right), \\
u_{k}(0)=u_{0 k}, \quad u_{k, t}(0)=u_{1 k}, \quad j=1,2, \ldots, k
\end{array}\right.
$$

and, as $k \rightarrow \infty$,

$$
u_{0 k}=\sum_{j=1}^{k}\left(u_{0}, \omega_{j}\right) \omega_{j} \rightarrow u_{0} \quad \text { in } H_{0}^{1} \quad \text { and } \quad u_{1 k}=\sum_{j=1}^{k}\left(u_{1}, \omega_{j}\right) \omega_{j} \rightarrow u_{1} \quad \text { in } H_{0}^{1}
$$

Here we denote by $(\cdot, \cdot)$ the inner product in $L^{2}(\Omega)$. Then $(3.1)$ can be reduced to the second-order ODE system

$$
\left\{\begin{array}{l}
\left(\left|\sum_{i=1}^{k} c_{k}^{i^{\prime}}(t) \omega_{i}\right|^{\rho} \sum_{i=1}^{k} c_{k}^{i^{\prime \prime}}(t) \omega_{i}, \omega_{j}\right)+\lambda_{j} c_{k}^{j}(t)+\lambda_{j} c_{k}^{j^{\prime \prime}}(t)+\gamma \lambda_{j} c_{k}^{j^{\prime}}(t) \\
\quad+\lambda_{j} \int_{0}^{\infty} \mu(\tau)\left(c_{k}^{j}(t)-c_{k}^{j}(t-\tau)\right) d \tau=\left(f\left(\sum_{j=1}^{k} c_{k}^{j}(t) \omega_{j}\right), \omega_{j}\right), \\
c_{k}^{j}(0)=\left(u_{0}, \omega_{j}\right), \quad c_{k}^{j^{\prime}}(0)=\left(u_{1}, \omega_{j}\right), \quad j=1,2, \ldots, k,
\end{array}\right.
$$

According to the standard existence theory for ordinary differential equations, we infer that system (3.3) admits a solution $c_{k}^{j}(t)$ in $\left[0, t_{m}\right)$, where $t_{m}>0$. Then we can obtain an approximate solution $u_{k}(t)$ of $(3.1)$ in $V_{k}$ over $\left[0, t_{m}\right)$, and the solution can be extended to $[0, T]$ for any given $T>0$. 
Multiplying (3.3) by $c_{k}^{j^{\prime}}(t)$ and summing with respect to $j$, we conclude that

$$
\begin{aligned}
& \frac{d}{d t}\left(\frac{1}{\rho+2}\left\|u_{k t}\right\|_{\rho+2}^{\rho+2}+\frac{1}{2}\left\|\nabla u_{k}\right\|_{2}^{2}+\frac{1}{2}\left\|\nabla u_{k t}\right\|_{2}^{2}\right)+\gamma\left\|\nabla u_{k t}\right\|_{2}^{2}+\int_{0}^{\infty} \mu(\tau)\left(\nabla \eta_{k}, \nabla u_{k t}\right) d \tau \\
& \quad=\left(f\left(u_{k}\right), u_{k t}^{\prime}\right) .
\end{aligned}
$$

Simple calculations yield

$$
\begin{aligned}
\int_{0}^{\infty} \mu(\tau)\left(\nabla \eta_{k}, \nabla u_{k t}\right) d \tau \\
=\int_{0}^{\infty} \mu(\tau)\left(\nabla \eta_{k}, \nabla \eta_{k t}+\nabla \eta_{k \tau}\right) d \tau=\frac{1}{2} \frac{d}{d t}\left\|\eta_{k}\right\|_{\mathcal{M}}^{2}+\left(\nabla \eta_{\tau}, \nabla \eta\right)_{\mathcal{M}} \\
\left(\eta_{k \tau}, \eta_{k}\right)_{\mathcal{M}}=\frac{1}{2} \int_{\Omega}\left(\int_{0}^{\infty} \mu(\tau) \frac{\partial}{\partial \tau}\left|\nabla \eta_{k}\right|^{2} d \tau\right) d x \\
=-\frac{1}{2} \int_{\Omega}\left(\int_{0}^{\infty} \mu^{\prime}(\tau)\left|\nabla \eta_{k}\right|^{2} d \tau\right) d x \\
=-\frac{1}{2} \int_{0}^{\infty} \mu^{\prime}(\tau)\left\|\nabla \eta_{k}\right\|_{2}^{2} d \tau .
\end{aligned}
$$

Combining (3.4) and (3.6), we find

$$
\begin{aligned}
& \frac{d}{d t}\left(\frac{1}{\rho+2}\left\|u_{k t}\right\|_{\rho+2}^{\rho+2}+\frac{1}{2}\left\|\nabla u_{k}\right\|_{2}^{2}+\frac{1}{2}\left\|\nabla u_{k t}\right\|_{2}^{2}+\frac{1}{2}\left\|\eta_{k}\right\|_{\mathcal{M}}^{2}-\int_{\Omega} F\left(u_{k}\right) d x\right) \\
& \quad=-\gamma\left\|\nabla u_{k t}\right\|_{2}^{2}+\frac{1}{2} \int_{0}^{\infty} \mu^{\prime}(\tau)\left\|\nabla_{k} \eta\right\|_{2}^{2} d \tau \leq 0 .
\end{aligned}
$$

Integrating (3.7) over $(0, t)$ and noting (3.2), we obtain

$$
\begin{aligned}
& \frac{1}{\rho+2}\left\|u_{k t}\right\|_{\rho+2}^{\rho+2}+\frac{1}{2}\left\|\nabla u_{k}\right\|_{2}^{2}+\frac{1}{2}\left\|\nabla u_{k t}\right\|_{2}^{2}+\frac{1}{2}\left\|\eta_{k}\right\|_{\mathcal{M}}^{2}-\int_{\Omega} F\left(u_{k}\right) d x \\
& \leq \frac{1}{\rho+2}\left\|u_{k t}(0)\right\|_{\rho+2}^{\rho+2}+\frac{1}{2}\left\|\nabla u_{k}(0)\right\|_{2}^{2}+\frac{1}{2}\left\|\nabla u_{k t}(0)\right\|_{2}^{2} \\
& \quad+\frac{1}{2}\left\|\eta_{k}(0)\right\|_{\mathcal{M}}^{2}-\int_{\Omega} F\left(u_{k}(0)\right) d x \\
& \leq K_{1}
\end{aligned}
$$

where $K_{1}$ is a constant independent of $k$. It follows from (3.8) and the Poincaré inequality that

$$
\left\{\begin{array}{l}
\left\{u_{k}\right\} \text { is bounded in } L^{\infty}\left(0, T ; H_{0}^{1}\right), \\
\left\{u_{k t}\right\} \text { is bounded in } L^{\infty}\left(0, T ; H_{0}^{1}\right), \\
\left\{\eta_{k t}\right\} \text { is bounded in } L^{2}(0, T ; \mathcal{M}) .
\end{array}\right.
$$


Multiplying (3.1) by $c_{k}^{j \prime}(t)$ and summing with respect to $j$, we obtain

$$
\begin{gathered}
\int_{\Omega}\left|u_{k t}\right|^{\rho}\left|u_{k t t}\right|^{2} d x+\left(\nabla u_{k}, \nabla u_{k t t}\right)+\left\|\nabla u_{k t t}\right\|_{2}^{2}+\frac{\gamma}{2} \frac{d}{d t}\left\|\nabla u_{k t}\right\|_{2}^{2} \\
+\int_{0}^{\infty} \mu(\tau)\left(\nabla \eta_{k}, \nabla u_{k t t}\right) d \tau=\left(f\left(u_{k}\right), u_{k t t}\right)
\end{gathered}
$$

that is,

$$
\begin{aligned}
& \int_{\Omega}\left|u_{k t}\right|^{\rho}\left|u_{k t t}\right|^{2} d x+\left\|\nabla u_{k t t}\right\|_{2}^{2}+\frac{\gamma}{2} \frac{d}{d t}\left\|\nabla u_{k t}\right\|_{2}^{2} \\
& =-\left(\nabla u_{k}, \nabla u_{k t t}\right)-\int_{0}^{\infty} \mu(\tau)\left(\nabla \eta_{k}, \nabla u_{k t t}\right) d \tau+\left(f\left(u_{k}\right), u_{k t t}\right)
\end{aligned}
$$

The right-hand side of (3.10) can be estimated as follows:

$$
\begin{aligned}
& \left|-\left(\nabla u_{k}, \nabla u_{k t t}\right)\right|=\left|-\int_{\Omega} \nabla u_{k} \nabla u_{k t t} d x\right| \leq \varepsilon\left\|\nabla u_{k t t}\right\|_{2}^{2}+\frac{1}{4 \varepsilon}\left\|\nabla u_{k}\right\|_{2}^{2} \quad \forall \varepsilon>0 \\
& \left|-\int_{0}^{\infty} \mu(\tau)\left(\nabla \eta_{k}, \nabla u_{k t t}\right) d \tau\right| \\
& \quad \leq \varepsilon\left\|\nabla u_{k t t}\right\|_{2}^{2}+\frac{1}{4 \varepsilon} \int_{\Omega}\left(\int_{0}^{\infty} \mu(\tau)\left|\nabla \eta_{k}\right| d \tau\right)^{2} d x \\
& \quad \leq \varepsilon\left\|\nabla u_{k t t}\right\|_{2}^{2}+\frac{1}{4 \varepsilon} \int_{\Omega}\left(\int_{0}^{\infty} \sqrt{\mu(\tau)} \sqrt{\mu(\tau)}\left|\nabla \eta_{k}\right| d \tau\right)^{2} d x \\
& \quad \leq \varepsilon\left\|\nabla u_{k t t}\right\|_{2}^{2}+\frac{1}{4 \varepsilon} \int_{0}^{\infty} \mu(\tau) d \tau \int_{\Omega} \int_{0}^{\infty} \mu(\tau)\left|\nabla \eta_{k}\right|^{2} d \tau d x \\
& \quad=\varepsilon\left\|\nabla u_{k t t}\right\|_{2}^{2}+\frac{k_{0}}{4 \varepsilon}\left\|\nabla \eta_{k}\right\|_{\mathcal{M}}^{2}
\end{aligned}
$$

with $k_{0}=\int_{0}^{\infty} \mu(\tau) d \tau$. Using $\left(A_{2}\right)$, the Sobolev embedding theorem, and the Poincaré inequality, we have

$$
\begin{aligned}
\left|\left(f\left(u_{k}\right), u_{k t t}\right)\right| & =\left|-\int_{\Omega} f\left(u_{k}\right) u_{k t t} d x\right| \\
& \leq c_{0} \int_{\Omega}\left(1+\left|u_{k}\right|^{p}\right)\left|u_{k}\right|\left|u_{k t t}\right| d x \\
& \leq C_{\varepsilon}\left(\left\|u_{k}\right\|_{2}^{2}+\left\|u_{k}\right\|_{2(p+1)}^{2(p+1)}\right)+\varepsilon\left\|u_{k t t}\right\|_{2}^{2} \\
& \leq C^{*}\left(\left\|\nabla u_{k}\right\|_{2}^{2}+\left\|\nabla u_{k}\right\|_{2}^{2(p+1)}\right)+\varepsilon C\left\|\nabla u_{k t t}\right\|_{2}^{2} .
\end{aligned}
$$

By (3.10)-(3.13) we have

$$
\begin{aligned}
& \int_{\Omega}\left|u_{k t}\right|^{\rho}\left|u_{k t t}\right|^{2} d x+\left\|\nabla u_{k t t}\right\|_{2}^{2}+\frac{\gamma}{2} \frac{d}{d t}\left\|\nabla u_{k t}\right\|_{2}^{2} \\
& \leq(2+C) \varepsilon\left\|\nabla u_{k t t}\right\|_{2}^{2}+\left(\frac{1}{4 \varepsilon}+C^{*}\right)\left\|\nabla u_{k}\right\|_{2}^{2}+C^{*}\left\|\nabla u_{k}\right\|_{2}^{2(p+1)}+\frac{k_{0}}{4 \varepsilon}\left\|\nabla \eta_{k}\right\|_{\mathcal{M}}^{2},
\end{aligned}
$$


that is,

$$
\begin{gathered}
\int_{\Omega}\left|u_{k t}\right|^{\rho}\left|u_{k t t}\right|^{2} d x+[1-(2+C) \varepsilon]\left\|\nabla u_{k t t}\right\|_{2}^{2}+\frac{\gamma}{2} \frac{d}{d t}\left\|\nabla u_{k t}\right\|_{2}^{2} \\
\leq\left(\frac{1}{4 \varepsilon}+C^{*}\right)\left\|\nabla u_{k}\right\|_{2}^{2}+C^{*}\left\|\nabla u_{k}\right\|_{2}^{2(p+1)}+\frac{k_{0}}{4 \varepsilon}\left\|\nabla \eta_{k}\right\|_{\mathcal{M}}^{2} .
\end{gathered}
$$

From (3.14) and (3.18) we know that

$$
\int_{\Omega}\left|u_{k t}\right|^{\rho}\left|u_{k t t}\right|^{2} d x+[1-(2+C) \varepsilon]\left\|\nabla u_{k t t}\right\|_{2}^{2}+\frac{\gamma}{2} \frac{d}{d t}\left\|\nabla u_{k t}\right\|_{2}^{2} \leq K_{2}
$$

Integrating (3.15) over $(0, t)(0<t \leq T)$ and noting (3.2) yield

$$
\int_{0}^{t} \int_{\Omega}\left|u_{k t t}\right|^{\rho}\left|u_{k t t}\right|^{2} d x d \tau+[1-(2+C) \varepsilon] \int_{0}^{t}\left\|\nabla u_{k t t}\right\|_{2}^{2} d \tau+\frac{\gamma}{2}\left\|\nabla u_{k t}\right\|_{2}^{2} \leq C_{T} .
$$

Taking $\varepsilon$ suitably small in (3.16), we can obtain the second estimate

$$
\int_{0}^{t}\left\|\nabla u_{k t t}\right\|_{2}^{2} d \tau+\left\|\nabla u_{k t}\right\|_{2}^{2} \leq C_{T}
$$

which implies that

$$
\left\{u_{k t t}\right\} \text { is uniformly bounded in } L^{2}\left(0, T ; H_{0}^{1}\right) .
$$

According to estimates (3.9) and (3.17), we infer that there exists a subsequence in $\left\{u_{m}\right\}$ (denoted by the same symbol) such that

$$
\left\{\begin{array}{l}
u_{k} \stackrel{*}{\rightarrow} u \quad \text { weak-star in } L^{\infty}\left(0, T ; H_{0}^{1}(\Omega)\right), \\
u_{k t} \stackrel{*}{\rightarrow} u_{t} \quad \text { weak-star in } L^{\infty}\left(0, T ; H_{0}^{1}(\Omega)\right), \\
u_{k t t} \stackrel{*}{\rightarrow} u_{t t} \quad \text { weakly in } L^{2}\left(0, T ; H_{0}^{1}(\Omega)\right), \\
\eta_{k} \stackrel{*}{\rightarrow} \eta \quad \text { weakly in } L^{2}(0, T ; \mathcal{M}),
\end{array}\right.
$$

which, combined with the Aubin-Lions compactness lemma, implies

$$
\left\{\begin{array}{l}
u_{k} \rightarrow u \quad \text { strongly in } C\left([0, T] ; H_{0}^{1}(\Omega)\right), \\
u_{k t} \rightarrow u_{t} \quad \text { strongly in } C\left([0, T] ; H_{0}^{1}(\Omega)\right) .
\end{array}\right.
$$

Using $\left(A_{2}\right)$ and (3.19), we get

$$
f\left(u_{k}\right) \rightarrow f(u) .
$$

From (3.19) we get $u_{k t} \rightarrow u_{t}$ a.e. in $\Omega \times(0, T)$. Hence

$$
\left|u_{k t}\right|^{\rho} u_{k t} \rightarrow\left|u_{t}\right|^{\rho} u_{t} \quad \text { a.e. in } \Omega \times(0, T) .
$$


On the other hand, by the Sobolev embedding theorem and $\left\|\nabla u_{k t}\right\|_{2}^{2} \leq L_{1}$, we have

$$
\begin{aligned}
\left\|\left|u_{k t}\right|^{\rho} u_{k t}\right\|_{L^{2}\left(0, T ; L^{2}(\Omega)\right)} & =\int_{0}^{T} \int_{\Omega}\left|u_{k t}\right|^{2(\rho+1)} d x d t \leq B^{2(\rho+1)} \int_{0}^{T}\left\|\nabla u_{k t}\right\|_{2}^{2(\rho+1)} d t \\
& \leq B^{2(\rho+1)} L_{1}^{(\rho+1)} T \leq C_{T} .
\end{aligned}
$$

Thus, using (3.20), (3.21), and the Lions lemma, we derive

$$
\left|u_{k t}\right|^{\rho} u_{k t} \rightarrow\left|u_{t}\right|^{\rho} u_{t} \quad \text { weakly in } L^{2}\left(0, T ; L^{2}(\Omega)\right)
$$

Let $\mathcal{D}(0, T)$ be the space of $C^{\infty}$ functions with compact support in $(0, T)$. Multiplying (3.1) by $\theta(t) \in \mathcal{D}(0, T)$ and integrating over $(0, T)$, it follows that

$$
\begin{aligned}
& \int_{0}^{T}\left(\left|u_{k t}\right|^{\rho} u_{k t t}, \omega_{j}\right) \theta(t) d t+\int_{0}^{T}\left(\nabla u_{k}, \nabla \omega_{j}\right) \theta(t) d t+\int_{0}^{T}\left(\nabla u_{k t t}, \nabla \omega_{j}\right) \theta(t) d t \\
& \quad+\gamma \int_{0}^{T}\left(\nabla u_{k t}, \nabla \omega_{j}\right) \theta(t) d t+\int_{0}^{T} \int_{0}^{\infty} \mu(\tau)\left(\nabla \eta_{k}, \nabla \omega_{j}\right) \theta(t) d t d \tau \\
& \quad=\int_{0}^{T}\left(f\left(u_{k}\right), \omega_{j}\right) \theta(t) d t .
\end{aligned}
$$

Noting that $\left\{\omega_{j}\right\}_{j=1}^{\infty}$ is a basis of $H_{0}^{1}$, via convergences (3.18), (3.19), and (3.22), we can get from (3.23) that

$$
\begin{aligned}
& \left(\left|u_{t}\right|^{\rho} u_{t t}, \omega_{j}\right)+\left(\nabla u, \nabla \omega_{j}\right)+\left(\nabla u_{t t}, \nabla \omega_{j}\right)+\gamma\left(\nabla u_{t}, \nabla \omega_{j}\right)+\int_{0}^{\infty} \mu(\tau)\left(\nabla \eta(\tau), \nabla \omega_{j}\right) d \tau \\
& \quad=\left(f(u), \omega_{j}\right),
\end{aligned}
$$

and hence, for all $\omega \in H_{0}^{1}(\Omega)$,

$$
\begin{aligned}
& \left(\left|u_{t}\right|^{\rho} u_{t t}, \omega\right)+(\nabla u, \nabla \omega)+\left(\nabla u_{t t}(t), \nabla \omega\right)+\gamma\left(\nabla u_{t}, \nabla \omega\right)+\int_{0}^{\infty} \mu(\tau)(\nabla \eta(\tau), \nabla \omega) d \tau \\
& \quad=(f(u), \omega) .
\end{aligned}
$$

Using (3.2) and (3.19), we have

$$
u(0)=u_{0}, \quad u_{t}(0)=u_{1} .
$$

On the other hand, by Pata and Zucchi [31] we have that

$$
\eta \in C([0, T], \mathcal{M})
$$

Combining (3.20), (3.25), and (3.26), we complete the proof.

Remark 2.4 For the uniqueness of the weak solution, see [1]. 


\section{Proof of Theorem 2.2}

Define the functionals

$$
\Phi(t)=\frac{1}{\rho+1} \int_{\Omega}\left|u_{t}\right|^{\rho} u_{t} u d x-\int_{\Omega} \Delta u_{t} u d x
$$

and

$$
\mathcal{L}(t)=M E(t)+\Phi(t),
$$

where $M>0$ will be fixed later. We recall that $E(t)$ is decreasing since $E^{\prime}(t) \leq 0$.

Lemma 4.1 For $M>0$ sufficiently large, there exist constants $\beta_{1}, \beta_{2}>0$ such that

$$
\beta_{1} E(t) \leq \mathcal{L}(t) \leq \beta_{2} E(t), \quad t \geq 0 .
$$

Proof By the Hölder and Cauchy inequalities we have

$$
\begin{aligned}
|\Phi(t)| \leq & \frac{1}{\rho+1} \int_{\Omega}\left|u_{t}\right|^{\rho+1}|u| d x+\left|\int_{\Omega} \nabla u_{t} \cdot \nabla u d x\right| \\
\leq & \frac{1}{\rho+1}\left(\int_{\Omega}\left|u_{t}\right|^{2(\rho+1)} d x\right)^{\frac{1}{2}}\left(\int_{\Omega}|u|^{2} d x\right)^{\frac{1}{2}} \\
& +\frac{1}{2}\left\|\nabla u_{t}\right\|_{2}^{2}+\frac{1}{2}\|\nabla u\|_{2}^{2} \\
= & \frac{1}{\rho+1}\left\|u_{t}\right\|_{2(\rho+1)}^{\rho+1}\|u\|_{2}+\frac{1}{2}\left\|\nabla u_{t}\right\|_{2}^{2}+\frac{1}{2}\|\nabla u\|_{2}^{2} .
\end{aligned}
$$

Since $E(t)$ is decreasing, from the Sobolev embedding theorem we have

$$
\begin{aligned}
\frac{1}{\rho+1}\left\|u_{t}\right\|_{2(\rho+1)}^{\rho+1}\|u\|_{2} & \leq \frac{1}{2(\rho+1)}\left\|u_{t}\right\|_{2(\rho+1)}^{2(\rho+1)}+\frac{1}{2(\rho+1)}\|u\|_{2}^{2} \\
& \leq C_{1} E^{\rho}(0)\left\|\nabla u_{t}\right\|_{2}^{2}+C_{1}\|\nabla u\|_{2}^{2} .
\end{aligned}
$$

Therefore

$$
|\Phi(t)| \leq C E(t) .
$$

Then taking $M>C$, we complete the proof.

Lemma 4.2 There exist $C_{2}>0$ and $C_{3}>0$, dependent on the initial data, such that

$$
\begin{gathered}
\Phi^{\prime}(t) \leq-E(t)-\left(\frac{1}{2}-\varepsilon\right)\|\nabla u(t)\|_{2}^{2}+C_{2}\left\|\nabla u_{t}(t)\right\|_{2}^{2} \\
-C_{3} \int_{0}^{\infty} \mu^{\prime}(\tau)\|\nabla \eta(\tau)\|_{2}^{2} d \tau \quad \forall t \geq 0 .
\end{gathered}
$$


Proof From the definition of $\Psi(t)$ we get

$$
\begin{aligned}
\Phi^{\prime}(t) & =\frac{1}{\rho+1} \int_{\Omega}\left(\left|u_{t}\right|^{\rho} u_{t t} u+\left|u_{t}\right|^{\rho} u_{t} u_{t}+\left(\left(u_{t}^{2}\right)^{\frac{\rho}{2}}\right)_{t} u_{t} u\right) d x-\int_{\Omega}\left(\Delta u_{t t} u+\Delta u_{t} u_{t}\right) d x \\
& =\frac{1}{\rho+1} \int_{\Omega}\left(\left|u_{t}(t)\right|^{\rho} u_{t t} u+\left|u_{t}\right|^{\rho} u_{t} u_{t}+\rho\left|u_{t}\right|^{\rho} u_{t t} u\right) d x-\int_{\Omega}\left(\Delta u_{t t} u+\left|\nabla u_{t}\right|^{2}\right) d x \\
& =\int_{\Omega}\left(\left|u_{t}\right|^{\rho} u_{t t}-\Delta u_{t t}\right) u d x+\frac{1}{\rho+1}\left\|u_{t}\right\|_{\rho+2}^{\rho+2}+\left\|\nabla u_{t}\right\|_{2}^{2} .
\end{aligned}
$$

Using (1.5), we easily see that

$$
\begin{aligned}
& \int_{\Omega}\left(\left|u_{t}\right|^{\rho} u_{t t}-\Delta u_{t t}\right) u d x \\
& \quad=-\|\nabla u\|_{2}^{2}+\int_{0}^{\infty} \mu(\tau)\left(\int_{\Omega} \Delta \eta(\tau) u(t) d x\right) d \tau+\gamma \int_{\Omega} \Delta u_{t} u d x+\int_{\Omega} f(u) u d x .
\end{aligned}
$$

We now estimate the second and third terms in the right-hand side as follows. Using the Cauchy inequality with $\varepsilon$ and the Hölder inequality, we have

$$
\begin{aligned}
& \left|\int_{0}^{\infty} \mu(\tau)\left(\int_{\Omega} \Delta \eta(\tau) u(t) d x\right) d \tau\right| \\
& \quad=\left|\int_{\Omega} \nabla u(t) \int_{0}^{\infty} \mu(\tau) \nabla \eta(\tau) d \tau d x\right| \\
& \quad \leq \frac{1}{2} \varepsilon\|\nabla u\|_{2}^{2}+\frac{1}{2 \varepsilon} \int_{\Omega}\left(\int_{0}^{\infty} \mu(\tau) \nabla \eta(\tau) d \tau\right)^{2} d x \\
& \quad \leq \frac{1}{2} \varepsilon\|\nabla u\|_{2}^{2}+\frac{k_{0}}{2 \varepsilon}\|\eta\|_{\mathcal{M}}^{2}
\end{aligned}
$$

and

$$
\left|\gamma \int_{\Omega} \Delta u_{t} u d x\right| \leq \frac{1}{2} \varepsilon\|\nabla u\|_{2}^{2}+\frac{\gamma^{2}}{2 \varepsilon}\left\|\nabla u_{t}\right\|_{2}^{2} .
$$

Therefore

$$
\Phi^{\prime}(t) \leq-(1-\varepsilon)\|\nabla u\|_{2}^{2}+\left(1+\frac{\gamma^{2}}{2 \varepsilon}\right)\left\|\nabla u_{t}\right\|_{2}^{2}+\frac{1}{\rho+1}\left\|u_{t}\right\|_{\rho+2}^{\rho+2}+\frac{k_{0}}{2 \varepsilon}\|\eta\|_{\mathcal{M}}^{2} .
$$

Noting the definitions of $E(t)$ and $\left(A_{2}\right)$, we obtain

$$
\begin{aligned}
\Phi^{\prime}(t) \leq & -E(t)-\left(\frac{1}{2}-\varepsilon\right)\|\nabla u\|_{2}^{2}+\left(\frac{3}{2}+\frac{\gamma^{2}}{2 \varepsilon}\right)\left\|\nabla u_{t}\right\|_{2}^{2}+\frac{2}{\rho+1}\left\|u_{t}\right\|_{\rho+2}^{\rho+2} \\
& +\left(\frac{1}{2} \frac{k_{0}}{2 \varepsilon}\right)\|\eta\|_{\mathcal{M}}^{2} .
\end{aligned}
$$

By Sobolev embedding we have

$$
\left\|u_{t}\right\|_{\rho+2}^{\rho+2} \leq B E(0)^{\frac{\rho}{2}}\left\|\nabla u_{t}\right\|_{2}^{2}
$$


Using $\left(A_{3}\right)$, we get

$$
\|\eta\|_{\mathcal{M}}^{2} \leq-\frac{1}{k_{1}} \int_{0}^{\infty} \mu^{\prime}(\tau)\|\nabla \eta(\tau)\|_{2}^{2} d \tau
$$

Combining (4.3)-(4.5), we finish the proof of Lemma 4.2.

Proof of Theorem 2.2 By Lemma 4.2 we have

$$
\Phi^{\prime}(t) \leq-E(t)+C_{2}\left\|\nabla u_{t}(t)\right\|_{2}^{2}-C_{3} \int_{0}^{\infty} \mu^{\prime}(\tau)\|\nabla \eta(\tau)\|_{2}^{2} d \tau
$$

Note that

$$
E^{\prime}(t)=-\gamma\left\|\nabla u_{t}(t)\right\|_{2}^{2}+\frac{1}{2} \int_{0}^{\infty} \mu^{\prime}(\tau)\|\nabla \eta(\tau)\|_{2}^{2} d \tau
$$

and

$$
\mathcal{L}(t)=M E(t)+\Phi(t)
$$

Then taking $M=\max \left(2 C_{3}, \frac{C_{2}}{\gamma}\right)$, we have

$$
\mathcal{L}^{\prime}(t) \leq-E(t)
$$

Using Lemma 4.1, we obtain

$$
\mathcal{L}^{\prime}(t) \leq-\frac{1}{\beta_{2}} \mathcal{L}(t)
$$

By the Gronwall inequality we obtain

$$
\mathcal{L}(t) \leq \mathcal{L}(0) e^{-\frac{1}{\beta_{2}} t}
$$

By Lemma 4.1 we have

$$
\beta_{1} E(t) \leq \mathcal{L}(t) \leq \beta_{2} E(0) e^{-\frac{1}{\beta_{2}} t}
$$

that is,

$$
E(t) \leq K e^{-v t}
$$

where $v=\frac{1}{\beta_{2}}$ and $K=\frac{\beta_{2} E(0)}{\beta_{1}}$.

\section{Proof of Theorem 2.3}

We define

$$
\begin{aligned}
& \Phi(t)=\frac{1}{\rho+1} \int_{\Omega}\left|u_{t}\right|^{\rho} u_{t} u d x-\int_{\Omega} \Delta u_{t} u d x \\
& \Psi(t)=\int_{\Omega} \Delta u_{t}\left(\int_{0}^{\infty} \mu(\tau) \eta d \tau\right) d x-\frac{1}{\rho+1} \int_{\Omega}\left|u_{t}\right|^{\rho} u_{t}\left(\int_{0}^{\infty} \mu(\tau) \eta d \tau\right) d x
\end{aligned}
$$


and set

$$
\mathcal{L}(t)=M E(t)+\varepsilon \Psi(t)+\chi(t),
$$

where $M$ and $\varepsilon$ will be fixed later.

Lemma 5.1 For $M>0$ sufficiently large, there exist constants $\beta_{1}, \beta_{2}>0$ such that

$$
\beta_{1} E(t) \leq \mathcal{L}(t) \leq \beta_{2} E(t), \quad t \geq 0,
$$

for any $0<\varepsilon \leq 1$.

Proof Since

$$
\begin{aligned}
\left|\int_{\Omega} \Delta u_{t}\left(\int_{0}^{\infty} \mu(\tau) \eta d \tau\right) d x\right| & \leq \int_{0}^{\infty} \mu(\tau)\left|\int_{\Omega} \Delta u_{t} \eta d x\right| d \tau \\
& =\int_{0}^{\infty} \mu(\tau)\left|\int_{\Omega} \nabla u_{t} \cdot \nabla \eta d x\right| d \tau \\
& \leq \frac{k_{0}}{2}\left\|\nabla u_{t}\right\|_{2}^{2}+\frac{1}{2}\|\eta\|_{\mathcal{M}}^{2},
\end{aligned}
$$

by the Hölder and Cauchy inequalities we have

$$
\begin{aligned}
\left.\frac{1}{\rho+1}\left|\int_{\Omega}\right| u_{t}\right|^{\rho} u_{t} \eta d x \mid & \leq \frac{1}{\rho+1} \int_{\Omega}\left|u_{t}(t)\right|^{\rho+1}|\eta| d x \\
& \leq \frac{1}{\rho+1}\left(\int_{\Omega}\left|u_{t}(t)\right|^{2(\rho+1)} d x\right)^{\frac{1}{2}}\left(\int_{\Omega}|\eta|^{2} d x\right)^{\frac{1}{2}} \\
& =\frac{1}{\rho+1}\left\|u_{t}\right\|_{2(\rho+1)}^{\rho+1}\|\eta\|_{2} .
\end{aligned}
$$

Since $E(t)$ is decreasing, from the Sobolev inequality we have

$$
\begin{aligned}
\frac{1}{\rho+1}\left\|u_{t}\right\|_{2(\rho+1)}^{\rho+1}\|\eta\|_{2} & \leq \frac{1}{2(\rho+1)}\left\|u_{t}\right\|_{2(\rho+1)}^{2(\rho+1)}+\frac{1}{2(\rho+1)}\|\eta\|_{2}^{2} \\
& \leq C_{1} E^{\rho}(0)\left\|\nabla u_{t}\right\|_{2}^{2}+C_{1}\|\nabla \eta\|_{2}^{2}
\end{aligned}
$$

Therefore

$$
\left.\left|-\frac{1}{\rho+1} \int_{\Omega}\right| u_{t}\right|^{\rho} u_{t}\left(\int_{0}^{\infty} \mu(\tau) \eta d \tau\right) d x \mid \leq k_{0} C_{1} E^{\rho}(0)\left\|\nabla u_{t}\right\|_{2}^{2}+C_{1}\|\nabla \eta\|_{\mathcal{M}}^{2} .
$$

Consequently,

$$
|\Psi(t)| \leq C E(t)
$$

Using Lemma 4.1 and taking $M$ large enough, we complete the proof of Lemma 5.1. 
Lemma 5.2 Under the conditions of Theorem 2.2, the functional

$$
\Phi(t)=\frac{1}{\rho+1} \int_{\Omega}\left|u_{t}(t)\right|^{\rho} u_{t}(t) u(t) d x-\int_{\Omega} \Delta u_{t}(t) u(t) d x
$$

satisfies

$$
\begin{aligned}
\Phi^{\prime}(t) \leq & -\left[1-\left(1+k_{0}\right) \delta_{1}\right]\|\nabla u\|_{2}^{2}+\left(1+\frac{\gamma^{2}}{4 \delta_{1}}\right)\left\|\nabla u_{t}\right\|_{2}^{2}+\frac{1}{\rho+1}\left\|u_{t}\right\|_{\rho+2}^{\rho+2} \\
& +\frac{1}{4 \delta_{1}} \int_{0}^{\infty} \mu^{2-q}(\tau) d \tau \int_{0}^{\infty} \mu^{q}(\tau)\|\nabla \eta\|_{2}^{2} d \tau+\int_{\Omega} f(u) u d x
\end{aligned}
$$

for any $\delta_{1}>0$.

Proof As in the proof of Lemma 4.2, we get

$$
\Phi^{\prime}(t)=\int_{\Omega}\left(\left|u_{t}\right|^{\rho} u_{t t}-\Delta u_{t t}\right) u d x+\frac{1}{\rho+1}\left\|u_{t}\right\|_{\rho+2}^{\rho+2}+\left\|\nabla u_{t}\right\|_{2}^{2}
$$

and

$$
\begin{aligned}
& \int_{\Omega}\left(\left|u_{t}\right|^{\rho} u_{t t}-\Delta u_{t t}\right) u d x \\
& =-\|\nabla u\|_{2}^{2}+\int_{0}^{\infty} \mu(\tau)\left(\int_{\Omega} \Delta \eta(\tau) u(t) d x\right) d \tau \\
& \quad+\gamma \int_{\Omega} \Delta u_{t} u d x+\int_{\Omega} f(u) u d x .
\end{aligned}
$$

By the Cauchy inequality the second and third terms can be estimated as follows:

$$
\begin{aligned}
& \int_{0}^{\infty} \mu(\tau)\left(\int_{\Omega} \Delta \eta u(t) d x\right) d \tau \\
& \quad \leq \delta_{1} k_{0}\|\nabla u\|_{2}^{2}+\frac{1}{4 \delta_{1}} \int_{0}^{\infty} \mu^{2-q}(\tau) d \tau \int_{0}^{\infty} \mu^{q}(\tau)\|\nabla \eta\|_{2}^{2} d \tau
\end{aligned}
$$

and

$$
\left|\gamma \int_{\Omega} \Delta u_{t} u d x\right|=\left|-\gamma \int_{\Omega} \nabla u_{t} \cdot \nabla u d x\right| \leq \delta_{1}\|\nabla u\|_{2}^{2}+\frac{\gamma^{2}}{4 \delta_{1}}\left\|\nabla u_{t}\right\|_{2}^{2},
$$

where $\delta_{1}>0$.

Combining (5.3)-(5.6), we establish Lemma 5.2.

Lemma 5.3 Under the conditions of Theorem 2.2, there exist constants $C, C^{\prime}, C^{\prime \prime}>0$ such that

$$
\Psi(t)=\int_{\Omega} \Delta u_{t}\left(\int_{0}^{\infty} \mu(\tau) \eta d \tau\right) d x-\frac{1}{\rho+1} \int_{\Omega}\left|u_{t}\right|^{\rho} u_{t}\left(\int_{0}^{\infty} \mu(\tau) \eta d \tau\right) d x
$$


satisfies

$$
\begin{aligned}
\Psi^{\prime}(t) \leq & \left(\delta_{2}+\delta_{2} C\right)\|\nabla u\|_{2}^{2}+\left(\delta_{2} \gamma^{2}-k_{0}+2 \delta_{2} C^{\prime}\right)\left\|\nabla u_{t}\right\|_{2}^{2}-\frac{k_{0}}{\rho+1}\left\|u_{t}\right\|_{\rho+2}^{\rho+2} \\
& +C^{\prime} \int_{0}^{\infty} \mu^{2-q}(\tau) d s \int_{0}^{\infty} \mu^{q}(\tau)\|\nabla \eta\|_{2}^{2} d \tau-2 C^{\prime \prime} \int_{0}^{\infty} \mu^{\prime}(\tau)\|\nabla \eta(\tau)\|_{2}^{2} d \tau
\end{aligned}
$$

for any $\delta_{2}>0$.

Proof From definition of $\Psi$ we have

$$
\begin{aligned}
\Psi^{\prime}(t)= & \int_{\Omega} \Delta u_{t t}\left(\int_{0}^{\infty} \mu(\tau) \eta d \tau\right) d x+\int_{\Omega} \Delta u_{t}\left(\int_{0}^{\infty} \mu(\tau) \eta_{t} d \tau\right) d x \\
& -\frac{1}{\rho+1} \int_{\Omega}\left|u_{t}\right|^{\rho} u_{t t}\left(\int_{0}^{\infty} \mu(\tau) \eta d \tau\right) d x \\
& -\frac{1}{\rho+1} \int_{\Omega}\left|u_{t}\right|^{\rho} u_{t}\left(\int_{0}^{\infty} \mu(\tau) \eta_{t} d \tau\right) d x \\
& -\frac{1}{\rho+1} \int_{\Omega}\left(\left(u_{t}^{2}\right)^{\frac{\rho}{2}}\right)_{t} u_{t}\left(\int_{0}^{\infty} \mu(\tau) \eta d \tau\right) d x \\
= & \int_{\Omega}\left(-\left|u_{t}\right|^{\rho} u_{t t}+\Delta u_{t t}\right)\left(\int_{0}^{\infty} \mu(\tau) \eta d \tau\right) d x \\
& +\int_{\Omega}\left(-\frac{\left|u_{t}\right|^{\rho} u_{t}}{\rho+1}+\Delta u_{t}\right)\left(\int_{0}^{\infty} \mu(\tau) \eta_{t} d \tau\right) d x \\
:= & I_{1}+I_{2} .
\end{aligned}
$$

From (1.5) we see that

$$
I_{1}=\int_{\Omega}\left(-\Delta u-\int_{0}^{\infty} \mu(\tau) \Delta \eta d \tau-\gamma \Delta u_{t}-f(u)\right)\left(\int_{0}^{\infty} \mu(\tau) \eta d \tau\right) d x .
$$

By the Green formula and the Cauchy and Hölder inequalities we have the following estimates:

$$
\begin{aligned}
& \int_{\Omega}-\Delta u\left(\int_{0}^{\infty} \mu(\tau) \eta d \tau\right) d x \\
& \quad=\int_{\Omega} \nabla u \cdot\left(\int_{0}^{\infty} \mu(\tau) \nabla \eta d \tau\right) d x \\
& \quad \leq \delta_{2}\|\nabla u\|_{2}^{2}+\frac{1}{4 \delta_{2}} \int_{0}^{\infty} \mu^{2-q}(\tau) d \tau \int_{0}^{\infty} \mu^{q}(\tau)\|\nabla \eta\|_{2}^{2} d \tau, \\
& -\gamma \int_{\Omega} \Delta u_{t}\left(\int_{0}^{\infty} \mu(\tau) \eta(\tau) d \tau\right) d x \\
& \quad=\gamma \int_{\Omega} \nabla u_{t} \cdot\left(\int_{0}^{\infty} \mu(\tau) \nabla \eta d \tau\right) d x \\
& \quad \leq \delta_{2} \gamma^{2}\left\|\nabla u_{t}\right\|_{2}^{2}+\frac{1}{4 \delta_{2}} \int_{0}^{\infty} \mu^{2-q}(\tau) d \tau \int_{0}^{\infty} \mu^{q}(\tau)\|\nabla \eta\|_{2}^{2} d \tau,
\end{aligned}
$$


and

$$
\begin{aligned}
-\int_{\Omega}\left(\int_{0}^{\infty} \mu(\tau) \Delta \eta d \tau\right)\left(\int_{0}^{\infty} \mu(\tau) \eta d \tau\right) d x & =\int_{\Omega}\left(\int_{0}^{\infty} \mu^{\frac{2-q}{2}} \mu^{\frac{q}{2}}(\tau) \nabla \eta(\tau) d \tau\right)^{2} d x \\
& \leq \int_{0}^{\infty} \mu^{2-q}(\tau) d \tau \int_{0}^{\infty} \mu^{q}(\tau)\|\nabla \eta\|_{2}^{2} d \tau .
\end{aligned}
$$

Using $\left(A_{2}\right)$ and the Cauchy, Hölder, and Poincaré inequalities, we obtain

$$
-\int_{\Omega} f(u)\left(\int_{0}^{\infty} \mu(\tau) \eta d \tau\right) d x \leq \delta_{2} C\|\nabla u\|_{2}^{2}+\frac{1}{4 \delta_{2}} \int_{0}^{\infty} \mu^{2-q}(\tau) d \tau \int_{0}^{\infty} \mu^{q}(\tau)\|\nabla \eta\|_{2}^{2} d \tau .
$$

Therefore

$$
I_{1} \leq\left(\delta_{2}+\delta_{2} C\right)\|\nabla u\|_{2}^{2}+\delta_{2} \gamma_{2}\left\|\nabla u_{t}\right\|_{2}^{2}+C^{\prime} \int_{0}^{\infty} \mu^{2-q}(\tau) d \tau \int_{0}^{\infty} \mu^{q}(\tau)\|\nabla \eta\|_{2}^{2} d \tau
$$

From (1.5) we easily obtain

$$
\int_{0}^{\infty} \mu(\tau) \eta_{t} d \tau=-\int_{0}^{\infty} \mu(\tau) \eta_{\tau} d \tau+\int_{0}^{\infty} \mu(\tau) u_{t}(t) d \tau=\int_{0}^{\infty} \mu^{\prime}(\tau) \eta d \tau+k_{0} u_{t}(t) .
$$

Then

$$
\begin{aligned}
I_{2}= & -k_{0}\left\|\nabla u_{t}(t)\right\|_{2}^{2}-\frac{k_{0}}{\rho+1}\left\|u_{t}\right\|_{\rho+2}^{\rho+2}+\int_{0}^{\infty} \mu^{\prime}(\tau)\left(\int_{\Omega} \Delta u_{t}(t) \eta d x\right) d \tau \\
& +\frac{1}{\rho+1} \int_{0}^{\infty} \mu^{\prime}(\tau)\left(\int_{\Omega}-\left|u_{t}(t)\right|^{\rho} u_{t}(t) \eta d x\right) d \tau .
\end{aligned}
$$

Using the Green formula and the Cauchy and Hölder inequalities, we have

$$
\begin{aligned}
\int_{0}^{\infty} \mu^{\prime}(\tau)\left(\int_{\Omega} \Delta u_{t}(t) \eta d x\right) d \tau & =-\int_{0}^{\infty} \mu^{\prime}(\tau)\left(\int_{\Omega} \nabla u_{t}(t) \cdot \nabla \eta d x\right) d \tau \\
& \leq-\int_{0}^{\infty} \mu^{\prime}(\tau)\left\|\nabla u_{t}\right\|_{2}\|\nabla \eta\|_{2} d \tau \\
& \leq \delta_{2} C^{\prime}\left\|\nabla u_{t}\right\|_{2}^{2}-C^{\prime \prime} \int_{0}^{\infty} \mu^{\prime}(\tau)\|\nabla \eta\|_{2}^{2} d \tau
\end{aligned}
$$

Using the method similar to that in the proof of Lemma 4.1, we get

$$
\frac{1}{\rho+1} \int_{0}^{\infty} \mu^{\prime}(\tau)\left(\int_{\Omega}-\left|u_{t}(t)\right|^{\rho} u_{t}(t) \eta d x\right) d \tau \leq \delta_{2} C^{\prime}\left\|\nabla u_{t}\right\|_{2}^{2}-C^{\prime \prime} \int_{0}^{\infty} \mu^{\prime}(\tau)\|\nabla \eta\|_{2}^{2} d \tau
$$

Then

$$
I_{2} \leq\left(-k_{0}+2 \delta_{2} C^{\prime}\right)\left\|\nabla u_{t}\right\|_{2}^{2}-\frac{k_{0}}{\rho+1}\left\|u_{t}\right\|_{\rho+2}^{\rho+2}-2 C^{\prime \prime} \int_{0}^{\infty} \mu^{\prime}(\tau)\|\nabla \eta\|_{2}^{2} d \tau
$$

Considering (5.7) and (5.8), we arrive at the conclusion. 
Proof of Theorem 2.3 Using

$$
\begin{aligned}
& \mathcal{L}(t)=M E(t)+\varepsilon \Phi(t)+\Psi(t) \\
& E^{\prime}(t)=-\gamma\left\|\nabla u_{t}(t)\right\|_{2}^{2}+\frac{1}{2} \int_{0}^{\infty} \mu^{\prime}(\tau)\|\nabla \eta(\tau)\|_{2}^{2} d \tau \leq 0,
\end{aligned}
$$

and Lemmas 5.2-5.3, we get

$$
\begin{aligned}
\mathcal{L}^{\prime}(t)= & M E^{\prime}(t)+\varepsilon \Phi^{\prime}(t)+\Psi^{\prime}(t) \\
\leq & {\left[-\gamma M+\left(1+\frac{\gamma^{2}}{4 \delta_{1}}\right) \varepsilon+\gamma^{2} \delta_{2}-k_{0}+2 \delta_{2} C^{\prime}\right]\left\|\nabla u_{t}\right\|_{2}^{2} } \\
& +\left[-\left(1-\left(k_{0}+1\right) \delta_{1}\right) \varepsilon+\delta_{2}+\delta_{2} C\right]\|\nabla u\|_{2}^{2}-\left[\frac{k_{0}}{\rho+1}-\frac{\varepsilon}{\rho+1}\right]\left\|u_{t}\right\|_{\rho+2}^{\rho+2} \\
& +\left[\frac{M}{2}-2 C^{\prime \prime}\right] \int_{0}^{\infty} \mu^{\prime}(\tau)\|\nabla \eta\|_{2}^{2} d \tau \\
& +\left[\frac{\varepsilon}{4 \delta_{1}}+C^{\prime}\right] \int_{0}^{\infty} \mu^{2-q}(\tau) d \tau \int_{0}^{\infty} \mu^{q}(\tau)\|\nabla \eta\|_{2}^{2} d \tau+\varepsilon \int_{\Omega} f(u) u d x .
\end{aligned}
$$

Taking $M>0$ sufficiently large and suitable $\varepsilon, \delta_{1}, \delta_{2}>0$ such that

$$
\begin{aligned}
& \frac{k_{0}}{\rho+1}-\frac{\varepsilon}{\rho+1}>0, \quad-\left(1-2 \delta_{1}\right) \varepsilon+\delta_{2}+\delta_{2} C<0, \\
& -\gamma M+\left(1+\frac{\gamma^{2}}{4 \delta_{1}}\right) \varepsilon+\gamma^{2} \delta_{2}-k_{0}+2 \delta_{2} C^{\prime}<0, \quad \frac{M}{2}-2 C^{\prime \prime}>0,
\end{aligned}
$$

by using the inequality $\mu^{\prime}(\tau) \leq-k_{1} \mu^{q}(\tau)$ we have

$$
\left(\frac{M}{2}-2 C^{\prime \prime}\right) \int_{0}^{\infty} \mu^{\prime}(\tau)\|\nabla \eta\|_{2}^{2} d \tau \leq-k_{1}\left(\frac{M}{2}-2 C^{\prime \prime}\right) \int_{0}^{\infty} \mu^{q}(\tau)\|\nabla \eta\|_{2}^{2} d \tau .
$$

Therefore

$$
\begin{aligned}
\mathcal{L}^{\prime}(t) \leq & {\left[-\gamma M+\left(1+\frac{\gamma^{2}}{4 \delta_{1}}\right) \varepsilon+\gamma^{2} \delta_{2}-k_{0}+2 \delta_{2} C^{\prime}\right]\left\|\nabla u_{t}\right\|_{2}^{2} } \\
& +\left[-\left(1-\left(k_{0}+1\right) \delta_{1}\right) \varepsilon+\delta_{2}+\delta_{2} C\right]\|\nabla u\|_{2}^{2}-\left[\frac{k_{0}}{\rho+1}-\frac{\varepsilon}{\rho+1}\right]\left\|u_{t}\right\|_{\rho+2}^{\rho+2} \\
& -\left[k_{1}\left(\frac{M}{2}-2 C^{\prime \prime}\right)-\left(\frac{\varepsilon}{4 \delta_{1}}+C^{\prime}\right) \int_{0}^{\infty} \mu^{2-q}(\tau) d \tau\right] \int_{0}^{\infty} \mu^{q}(\tau)\|\nabla \eta\|_{2}^{2} d \tau \\
& +\varepsilon \int_{\Omega} f(u) u d x .
\end{aligned}
$$

By Remark 2.1 and $\left(A_{2}\right)$, taking suitable $M>0, \varepsilon, \delta_{1}, \delta_{2}>0$, we get

$$
\begin{aligned}
\mathcal{L}^{\prime}(t) & \leq-C\left(\left\|\nabla u_{t}\right\|_{2}^{2}+\|\nabla u\|_{2}^{2}+\left\|u_{t}\right\|_{\rho+2}^{\rho+2}+\int_{0}^{\infty} \mu^{q}(\tau)\|\nabla \eta\|_{2}^{2} d \tau-\int_{\Omega} f(u) u d x\right) \\
& \leq-C\left(\left\|\nabla u_{t}\right\|_{2}^{2}+\|\nabla u\|_{2}^{2}+\left\|u_{t}\right\|_{\rho+2}^{\rho+2}+\int_{0}^{\infty} \mu^{q}(\tau)\|\nabla \eta\|_{2}^{2} d \tau\right) .
\end{aligned}
$$


Using $\left(A_{3}\right)$, we can easily show that $\int_{0}^{\infty} \mu^{1-\theta}(\tau) d \tau<\infty$ for any $\theta<2-q$. Then

$$
\begin{aligned}
\Lambda & :=\int_{0}^{\infty} \mu^{1-\theta}(\tau) \int_{\Omega}|\nabla \eta|^{2} d x d \tau \\
& \leq 2 \int_{0}^{\infty} \mu^{1-\theta}(\tau) \int_{\Omega}\left(|\nabla u(t)|^{2}+|\nabla u(t-\tau)|^{2}\right) d x d \tau \\
& \leq C \int_{0}^{\infty} \mu^{1-\theta}(\tau) d \tau<L
\end{aligned}
$$

with positive constant $L>1$.

Using the conditions of Theorem 2.3, the Hölder inequality, and (5.10), we see that

$$
\begin{aligned}
\|\eta\|_{\mathcal{M}}^{2} & =\int_{0}^{\infty} \mu(\tau) \int_{\Omega}|\nabla \eta|^{2} d x d \tau=\int_{0}^{\infty} \mu(\tau)\|\nabla \eta\|_{2}^{2} d \tau \\
& =\int_{0}^{\infty} \mu^{\frac{(q-1)(1-\theta)}{q-1+\theta}}(\tau)\|\nabla \eta\|_{2}^{2 \frac{(q-1)}{q-1+\theta}} \cdot \mu^{\frac{\theta q}{q-1+\theta}}(\tau)\|\nabla \eta\|_{2}^{2 \frac{\theta}{q-1+\theta}} d \tau \\
& \leq\left[\int_{0}^{\infty} \mu^{1-\theta}(\tau)\|\nabla \eta\|_{2}^{2} d \tau\right]^{\frac{q-1}{q-1+\theta}} \cdot\left[\int_{0}^{\infty} \mu^{q}(\tau)\|\nabla \eta\|_{2}^{2} d \tau\right]^{\frac{\theta}{q-1+\theta}} \\
& \leq L\left[\int_{0}^{\infty} \mu^{q}(\tau)|\nabla \eta|_{2}^{2} d \tau\right]^{\frac{\theta}{q-1+\theta}} .
\end{aligned}
$$

Therefore we get, for $\sigma>1$,

$$
\begin{aligned}
E^{\sigma}(t) \leq & C E^{\sigma-1}(0)\left\{\left\|\nabla u_{t}\right\|_{2}^{2}+\left\|u_{t}\right\|_{\rho+2}^{\rho+2}+\|\nabla u\|_{2}^{2}-\int_{\Omega} F(u) d x\right\} \\
& +C\left(\int_{0}^{\infty} \mu(\tau)\|\nabla \eta\|_{2}^{2} d x d \tau\right)^{\sigma} \\
\leq & C E^{\sigma-1}(0)\left\{\left\|\nabla u_{t}\right\|_{2}^{2}+\left\|u_{t}\right\|_{\rho+2}^{\rho+2}+\|\nabla u\|_{2}^{2}-\int_{\Omega} F(u) d x\right\} \\
& +C\left\{\int_{0}^{\infty} \mu^{q}(\tau)\|\nabla \eta\|_{2}^{2} d \tau\right\}^{\frac{\sigma \theta}{q-1+\theta}} .
\end{aligned}
$$

By choosing $\theta=\frac{1}{2}$ and $\sigma=2 q-1$ (hence $\frac{\sigma \theta}{q-1+\theta}=1$ ) estimate (5.12) gives

$$
E^{\sigma}(t) \leq C\left\{\left\|\nabla u_{t}\right\|_{2}^{2}+\left\|u_{t}\right\|_{\rho+2}^{\rho+2}+\|\nabla u\|_{2}^{2}-\int_{\Omega} F(u) d x+\int_{0}^{\infty} \mu^{q}(\tau)\|\nabla \eta\|_{2}^{2} d \tau\right\} .
$$

A combination of (5.9) and (5.13) then leads to

$$
\mathcal{L}^{\prime}(t) \leq-C E^{\sigma}(t)
$$

By Lemma 5.1 we have

$$
\mathcal{L}^{\prime}(t) \leq-C \frac{1}{\beta_{2}^{\sigma}} \mathcal{L}^{\sigma}(t)
$$


A simple integration of (5.14) over $(0, t)$ yields

$$
\mathcal{L}(t) \leq C_{1}(1+t)^{-\frac{1}{\sigma-1}}, \quad t \geq 0
$$

that is,

$$
\mathcal{L}(t) \leq C_{1}(1+t)^{-\frac{1}{2(q-1)}}, \quad t \geq 0
$$

\section{Conclusions}

In this paper, we consider the Dirichlet boundary value problem of nonlinear evolution equation with hereditary memory, variable density, and external force term. We prove the existence of a global solution by means of the Galerkin method, establish the exponential stability by using only one auxiliary functional (this method is simpler than that in [1]), and also show the polynomial stability under suitable conditions. Under suitable hypotheses on the external force term function $f$ and integral kernel function $\mu$ with $\gamma \geq 0$ in the model, we can further consider the local existence and blowup phenomenon of the solution.

\section{Acknowledgements}

The authors would like to thank the referee for his/her careful reading and kind suggestions.

\section{Funding}

This work was supported by National Natural Science Foundation of China (No. 11201258).

\section{Availability of data and materials}

The data used to support the findings of this study are available from the corresponding author upon request.

\section{Competing interests}

The authors declare that they have no competing interests.

\section{Authors' contributions}

The authors contributed equally to the writing of this paper. The authors read and approved the final manuscript.

\section{Authors' information}

FL currently works at the School of Mathematical Sciences, Qufu Normal University, P.R. China. He does research in Applied Mathematics and Analysis. He and his group are engaged in the research on the well-posedness and longtime dynamics for some nonlinear evolution equations. ZJ has got Master's degree in Qufu Normal University and is a member of Li's group.

\section{Publisher's Note}

Springer Nature remains neutral with regard to jurisdictional claims in published maps and institutional affiliations.

Received: 18 November 2018 Accepted: 7 February 2019 Published online: 15 February 2019

\section{References}

1. Araújo, R.O., Ma, T.F., Qin, Y.: Long-time behavior of a quasilinear viscoelastic align with past history. J. Differ. Equ. 254, 4066-4087 (2013)

2. Cavalcanti, M.M., Domingos Cavalcanti, V.N., Ferreira, J.: Existence and uniform decay for a non-linear viscoelastic equation with strong damping. Math. Methods Appl. Sci. 24, 1043-1053 (2001)

3. Cavalcanti, M.M., Domingos Cavalcanti, V.N., Lasiecka, I., Webler, C.M.: Intrinsic decay rates for the energy of a nonlinear viscoelastic equation modeling the vibrations of thin rods with variable density. Adv. Nonlinear Anal. 6, 121-145 (2017)

4. Conti, M., Ma, T.F., Marchini, E.M., Seminario Huertas, P.N.: Asymptotics of viscoelastic materials with nonlinear density and memory effects. J. Differ. Equ. 264, 4235-4259 (2018)

5. Conti, M., Marchini, E.M., Pata, V.: A well posedness result for nonlinear viscoelastic equations with memory. Nonlinear Anal. 94, 206-216 (2014)

6. Du, G., Li, F.: Global higher integrability of solutions to subelliptic double obstacle problems. J. Appl. Anal. Comput. 8(3), 1021-1032 (2018)

7. Du, G., Li, F.: Interior regularity of obstacle problems for nonlinear subelliptic systems with VMO coefficients. J. Inequal. Appl. 2018, 53 (2018) 
8. Fabrizio, M., Morro, A.: Mathematical Problems in Linear Viscoelastic. SIAM Stud. Appl. Math. Society for Industrial and Applied Mathematics, Philadelphia (1992)

9. Fatori, L.H., Jorge Silva, M.A., Ma, T.F., Yang, Z.: Long-time behavior of a class of thermoelastic plates with nonlinear strain. J. Differ. Equ. 259, 4831-4862 (2015)

10. Gao, Q., Li, F., Wang, Y.: Blow-up of the solution for higher-order Kirchhoff-type equations with nonlinear dissipation. Cent. Eur. J. Math. 9(3), 686-698 (2011)

11. Han, X., Wang, M.: Global existence and uniform decay for a nonlinear viscoelastic equation with damping. Nonlinear Anal. 70, 3090-3098 (2009)

12. Han, X., Wang, M.: General decay of energy for a viscoelastic equation with nonlinear damping. Math. Methods Appl. Sci. 32(3), 346-358 (2009)

13. Li, F:: Global existence and uniqueness of weak solution to nonlinear viscoelastic full Marguerre-von Kármán shallow shell equations. Acta Math. Sin. 25, 2133-2156 (2009)

14. Li, F.: Limit behavior of the solution to nonlinear viscoelastic Marguerre-von Kármán shallow shell system. J. Differ. Equ. 249, 1241-1257 (2010)

15. Li, F., Bai, Y.: Uniform decay rates for nonlinear viscoelastic Marguerre-von Kármán equations. J. Math. Anal. Appl. 351 522-535 (2009)

16. Li, F., Bao, Y.: Uniform stability of the solution for a memory-type elasticity system with nonhomogeneous boundary control condition. J. Dyn. Control Syst. 23, 301-315 (2017)

17. Li, F., Du, G.: General energy decay for a degenerate viscoelastic Petrovsky-type plate equation with boundary feedback. J. Appl. Anal. Comput. 8(1), 390-401 (2018)

18. Li, F., Gao, Q.: Blow-up of solution for a nonlinear Petrovsky type equation with memory. Appl. Math. Comput. 274, 383-392 (2016)

19. Li, F., Hu, F.: Weighted integral inequality and applications in general energy decay estimate for a variable density wave equation with memory. Bound. Value Probl. 2018, 164 (2018)

20. Li, F., Li, J.: Global existence and blow-up phenomena for nonlinear divergence form parabolic equations with inhomogeneous Neumann boundary conditions. J. Math. Anal. Appl. 385, 1005-1014 (2012)

21. Li, F., Li, J.: Global existence and blow-up phenomena for $p$-Laplacian heat equation with inhomogeneous Neumann boundary conditions. Bound. Value Probl. 2014, 219 (2014)

22. Li, F., Zhao, C.: Uniform energy decay rates for nonlinear viscoelastic wave equation with nonlocal boundary damping. Nonlinear Anal. 74, 3468-3477 (2011)

23. Li, F., Zhao, Z., Chen, Y.: Global existence uniqueness and decay estimates for nonlinear viscoelastic wave equation with boundary dissipation. Nonlinear Anal., Real World Appl. 12, 1770-1784 (2011)

24. Liu, C., Peng, Y.: Stability of periodic steady-state solutions to a non-isentropic Euler-Maxwell system. Z. Angew. Math. Phys. 68, 105 (2017)

25. Liu, C.., Peng, Y.: Convergence of a non-isentropic Euler-Poisson system for all time. J. Math. Pures Appl. 119(9), 255-279 (2018)

26. Liu, W: General decay and blow-up of solution for a quasilinear viscoelastic problem with nonlinear source. Nonlinear Anal. 73, 1890-1904 (2010)

27. Love, A.E.H.: Treatise on Mathematical Theory of Elasticity. Dover, New York (1944)

28. Messaoudi, S.A., Tatar, N.: Global existence and uniform stability of solutions for a quasilinear viscoelastic problem. Math. Methods Appl. Sci. 30, 665-680 (2007)

29. Messaoudi, S.A., Tatar, N.: Exponential and polynomial decay for a quasilinear viscoelastic equation. Nonlinear Anal. $68,785-793(2008)$

30. Messaoudi, S.A., Tatar, N.: Exponential decay for a quasilinear viscoelastic equation. Math. Nachr. 282, 1443-1450 (2009)

31. Pata, V., Zucchi, A.: Attractors for a damped hyperbolic equation with linear memory. Adv. Math. Sci. Appl. 11 505-529 (2001)

32. Renardy, M., Hrusa, W.J., Nohel, J.A.: Mathematical Problems in Viscoelasticity. Pitman Monographs and Surveys in Pure and Applied Mathematics, vol. 35. Wiley, New York (1987)

\section{Submit your manuscript to a SpringerOpen ${ }^{\circ}$ journal and benefit from:}

- Convenient online submission

- Rigorous peer review

- Open access: articles freely available online

- High visibility within the field

Retaining the copyright to your article

Submit your next manuscript at $\boldsymbol{~ s p r i n g e r o p e n . c o m ~}$ 